\title{
Transverse beam compression on the Paul trap simulator experiment
}

\author{
Erik P. Gilson, ${ }^{*}$ Moses Chung, Ronald C. Davidson, Philip C. Efthimion, and Richard Majeski \\ Plasma Physics Laboratory, Princeton University, Princeton, New Jersey 08543, USA
}

(Received 28 June 2007; published 6 December 2007)

\begin{abstract}
The Paul trap simulator experiment is a compact laboratory Paul trap that simulates a long, thin charged-particle bunch coasting through a kilometers-long magnetic alternating-gradient (AG) transport system by putting the physicist in the beam's frame of reference. The transverse dynamics of particles in both systems are described by similar equations, including all nonlinear space-charge effects. The timedependent quadrupolar electric fields created by the confinement electrodes of a linear Paul trap correspond to the axially dependent magnetic fields applied in the AG system. Results are presented for experiments in which the lattice period and strength are changed over the course of the experiment to transversely compress a beam with an initial depressed tune of 0.9 . Instantaneous and smooth changes are considered. Emphasis is placed on determining the conditions that minimize the emittance growth and the number of halo particles produced by the beam compression process. Both the results of particle-in-cell simulations performed with the warp code and envelope equation solutions agree well with the experimental data.
\end{abstract}

DOI: 10.1103/PhysRevSTAB.10.124201

PACS numbers: 52.59.Sa, 29.27.- a, 41.85.Ja, 52.27.Jt

\section{INTRODUCTION}

The transverse compression of intense charged-particle beams after acceleration and transport allows for the delivery of large numbers of particles and large amounts of energy to small target spot sizes in applications such as high energy and nuclear physics, ion-beam-driven high energy density physics, heavy ion fusion, and spallation neutron sources [1-6]. The transverse compression should ideally be executed over as few lattice periods of the transport system as possible in order to keep the overall system length, and cost, minimized. However, transverse compression cannot be applied so rapidly as to drastically reduce the beam quality, excite collective-mode oscillations, or generate unwanted halo particles [7-11].

The Paul trap simulator experiment (PTSX) is a compact and flexible laboratory facility that simulates the propagation of intense charged-particle beams over thousands of lattice periods through magnetic alternating-gradient (AG) quadrupole transport systems [12-20]. The simulation makes use of the isomorphism between the transverse equations of motion for particles in the two systems [12,21-23]. In the work described in this paper, the PTSX facility has been used to perform transverse plasma compression experiments in order to develop a better understanding of the physics of transverse compression of intense beams.

The PTSX device is a linear Paul trap [24] confining a one-component plasma of particles with charge $e_{b}$, where the $e_{b} \boldsymbol{E}_{\perp}^{\text {ext }}$ forces that the PTSX electrodes exert on the trapped plasma particles are analogous to the $e_{b} \boldsymbol{v}_{z} \times \boldsymbol{B}_{\perp}^{\text {ext }}$ forces that the AG system exert on the beam particles in the beam frame provided that long, coasting beams that are

*egilson@pppl.gov thin relative to the AG system magnet spacing are considered. Specifically, the amplitude and frequency of the voltage waveform applied to the PTSX electrodes correspond to the quadrupole magnet strength and lattice spacing in the AG system. In addition to the equivalence of the applied forces, the self-field forces in both systems can be described by scalar potentials that obey Poisson's equation. In Ref. [12], it was shown that the self-consistent transverse Hamiltonians and the resulting Vlasov equations for the AG system and the PTSX system are equivalent, neglecting end effects. Thus, the very good confinement properties of ions in PTSX and the arbitrary form of the voltage waveform applied to the confining electrodes make PTSX a useful laboratory facility in which to simulate transverse beam compression in an AG system.

\section{PTSX APPARATUS}

As shown in Fig. 1, the PTSX device is a linear Paul trap constructed from a $2.8 \mathrm{~m}$-long, $r_{w}=10 \mathrm{~cm}$-radius cylinder. The cylinder is divided into two $40 \mathrm{~cm}$-long end
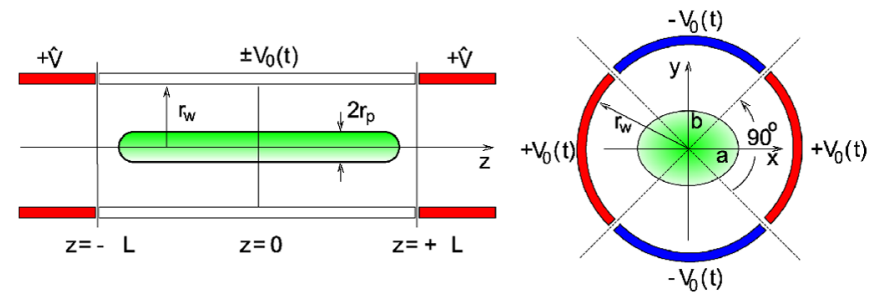

FIG. 1. (Color) The PTSX device consists of three cylindrical electrodes with radius $r_{w}=0.1 \mathrm{~m}$, each divided into four $90^{\circ}$ sectors. An oscillating voltage $\pm V_{0}(t)$ confines the charge bunch in the transverse plane to a radius $r_{p}$. Static voltages $+\hat{V}$ on the end electrodes confine the ions axially within a length $2 L=$ $2 \mathrm{~m}$. 
cylinders and a $2 L=2 \mathrm{~m}$-long central cylinder. All cylinders are azimuthally divided into four $90^{\circ}$ segments so that, when an oscillating voltage $V_{0}(t)$ is applied with alternating polarity on adjacent segments, the resulting oscillating transverse quadrupole electric field exerts a ponderomotive force that confines the plasma radially. To trap the plasma axially, the two end cylinders are biased to a constant voltage $\hat{V}$. Voltage waveforms with amplitudes up to $400 \mathrm{~V}$ and frequencies up to $100 \mathrm{kHz}$ can be used. The trapping voltage is nominally $\hat{V}=36 \mathrm{~V}$. The vacuum pressure of $5 \times 10^{-9}$ Torr prevents neutral collisions from playing an important role in the plasma behavior.

The plasma source is a $1.5 \mathrm{~cm}$-diameter aluminosilicate cesium emitter. Singly charged cesium ions are extracted by applying a bias of less than 10 volts between the emitter and an acceleration grid. The ions then pass through a separately biased deceleration grid. The ion source is situated in the middle of one of the $40 \mathrm{~cm}$-long cylinders, and to inject a pure cesium ion plasma into the trap, the segments on this $40 \mathrm{~cm}$-long cylinder are temporarily set to oscillate with the voltage $\pm V_{0}(t)$. The injection time $t_{i}$ is several milliseconds in order to allow cesium ions with several eV of kinetic energy to fill the trap. The injection process is optimized by briefly suppressing ion emission to allow ions to vacate the vicinity of the $40 \mathrm{~cm}$-long trapping electrodes to ensure that the trapped plasma is as quiescent and cool as possible [19].

After trapping the plasma for a time $t_{t}$, that can be up to $300 \mathrm{~ms}$ but is actually $1 \mathrm{~ms}$ for the experimental results presented here, the $40 \mathrm{~cm}$-long cylinder on the opposite end of the PTSX device from the ion source is set to oscillate with voltage $\pm V_{0}(t)$, and the plasma streams out of the trap. Part of the exiting plasma is collected on a movable $5 \mathrm{~mm}$-diameter collector disk. The inject-trapdump cycle is repeated to reduce the uncertainty in the data. The collector is moved along a null in the applied potential in the transverse plane in order to collect a radial charge profile of the trapped plasma. The radial density profile is then computed using the measured radial charge profile and knowledge of the area of the collector and the length of the plasma column [14]. Note that since the plasma ions can take several milliseconds to leave the trap, the measurements are necessarily averaged over hundreds of lattice periods.

\section{TRAPPED PLASMA PROPERTIES}

The circular cross section of the PTSX electrodes allows the time-dependent electric potential to be calculated analytically. Near the axis, the potential is quadrupolar and the average smooth-focusing frequency [1] of particles' transverse oscillations can be expressed for an applied voltage $V(t)=V_{0 \max } \sin (2 \pi f t)$ as $[1,12]$

$$
\omega_{q}=\frac{8 e_{b} V_{0 \max }}{m_{b} r_{w}^{2} \pi f} \xi
$$

where $m_{b}=133 \mathrm{amu}$ for $\mathrm{Cs}^{+}$ions in PTSX. The factor $\xi$ depends on the shape of the voltage waveform and $\xi=$ $1 / 2 \sqrt{2} \pi$ for the sinusoidal waveform used herein. Note that $\xi=4 \sqrt{3} /(\eta \sqrt{3-2} \eta)$ for a periodic step-function waveform with fill-factor $\eta$ (the so-called focusing-offdefocusing-off, or FODO lattice). In addition, the smooth-focusing vacuum phase advance $\sigma_{v}^{\text {sf }}$ is given by $\sigma_{v}^{\text {sf }}=\omega_{q} / f[1,13,15]$. In order for the particles to be confined radially, the normalized beam intensity $s \equiv$ $\omega_{p}^{2}(0) / 2 \omega_{q}^{2}$ must be less than unity, where $\omega_{p}(0)=$ $\left[n_{b}(0) e_{b}^{2} / m_{b} \epsilon_{0}\right]^{1 / 2}$ is the on-axis plasma frequency. The limit of low $s$ corresponds to the regime where the plasma's space-charge effects are small, while the limit $s \rightarrow 1$ corresponds to the limit of space-charge-dominated beams. For a flattop density profile, the normalized beam intensity parameter $s$ is related to the depressed tune $\nu / \nu_{o}$ as $\nu / \nu_{0}=\sqrt{1-s}$.

Under quasi-steady-state conditions, for a thermal equilibrium distribution of particles, the average density profile $n_{b}(r)$ is given by $[1,2]$

$$
n_{b}(r)=n_{b}(r=0) \exp \left[-\frac{m_{b} \omega_{q}^{2} r^{2}+2 e_{b} \phi^{s}(r)}{2 k T}\right] .
$$

Here, $k$ is Boltzmann's constant, $T=$ const is the effective transverse temperature, and the space-charge potential $\phi^{s}(r)$ is determined self-consistently from numerical integration of Poisson's equation $r^{-1} \partial_{r}\left(r \partial_{r} \phi^{s}\right)=$ $-n_{b}(r) e_{b} / \epsilon_{0}$. For $k T \rightarrow 0(s \rightarrow 1)$, the numerator in the exponential must also approach zero in order for the density to remain finite, and this implies a nearly uniformdensity plasma. In the case of low space-charge density $(s \rightarrow 0)$, the electrostatic potential term in the exponential can be neglected and the radial density profile is nearly Gaussian. As the normalized beam intensity $s$ varies between 0 and 1 , the radial density profile smoothly changes from a flattop distribution, to a bell-curve shape, and finally to a Gaussian. If $N_{b}=\int_{0}^{r_{w}} n_{b}(r) 2 \pi r d r$ is the line density, and $R_{b}^{2}=\left(1 / N_{b}\right) \int_{0}^{r_{w}} n_{b}(r) 2 \pi r^{3} d r$ is the mean-square radius of the plasma column, then the parameter $\zeta$, defined through the equation $N_{b}=n_{b}(0) \pi R_{b}^{2} \zeta$, is 1 when $s=0$ and $\zeta=2$ when $s=1$. For intermediate values of $s, \zeta$ must be determined from the numerically integrated solutions of Poisson's equation (see Fig. 2).

Integration of Eq. (2) over the radial distribution gives the global radial force balance equation [1],

$$
m_{b} \omega_{q}^{2} R_{b}^{2}=2 k T+\frac{N_{b} e_{b}^{2}}{4 \pi \epsilon_{0}}
$$

which states that the applied confining force must balance both the thermal pressure and the repulsive space-charge force. In the analysis of PTSX results, $R_{b}^{2}$ and $N_{b}$ are calculated as moments of the measured plasma density profiles; $k T$ is the only parameter not known a priori and is inferred from Eq. (3). The transverse emittance $\epsilon_{\perp}$ of a 


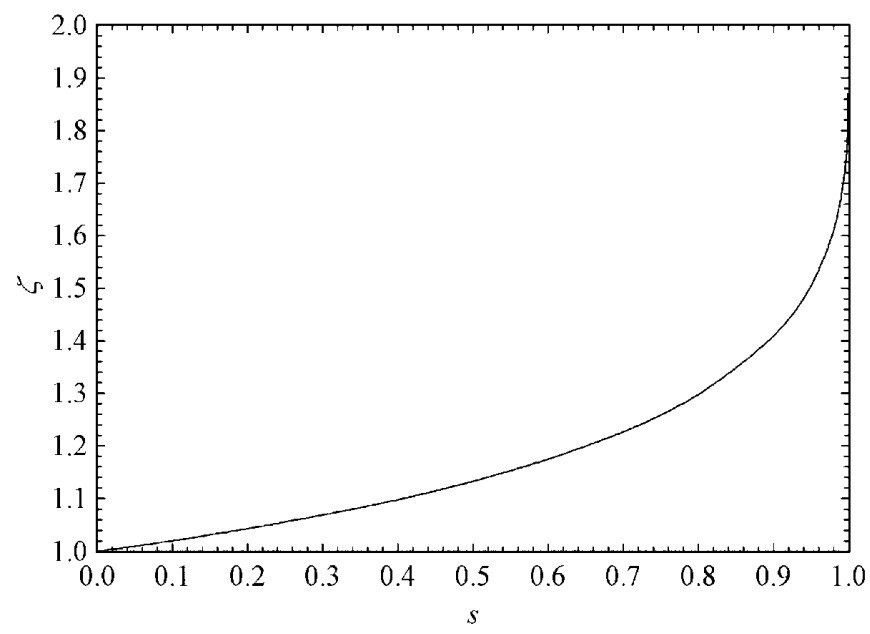

FIG. 2. The average radial density profile $n_{b}(r)$ is determined self-consistently from numerical integration of Poisson's equation and Eq. (2). The parameter $\zeta=N_{b} /\left[n_{b}(0) \pi R_{b}^{2}\right]$ is plotted versus the normalized beam intensity parameter $s$. The profile is Gaussian in the limit of weak space charge, $s \rightarrow 0$ and $\zeta=1$. The profile is uniform when $s \rightarrow 1$ and $\zeta=2$, corresponding to a space-charge-dominated beam.

beam is the phase-space area that the beam particle distribution occupies. Although the equivalent emittance of the trapped plasma in PTSX is not directly measured, relative changes in emittance can be inferred by noting that the emittance scales as $\epsilon_{\perp} \propto R_{b} \sqrt{k T\left(N_{b}, R_{b}\right)}$. Typical uncertainties in the emittance of $10 \%$ to $15 \%$ are due to the uncertainty in the computed value of $R_{b}^{2}$ that is, in turn, due to the amplification of small uncertainties in the measured radial density profile $n_{b}(r)$ of the tail ions by the measure $2 \pi r^{3} d r$.

\section{TRANSVERSE COMPRESSION}

Transverse compression of the beam can be achieved, in accordance with the global force balance equation [Eq. (3)], by increasing the transverse focusing frequency $\omega_{q}$ using the flexibility of the PTSX device to apply arbitrary voltage waveforms to the transverse confinement electrodes. Assuming that the line density $N_{b}$ does not change during the increase in $\omega_{q}$, the beam will be compressed as long as any increase in the effective perpendicular temperature $k T$ is not too great. Analytical and simulation work by Dorf et al. has explored the effects of adiabatic changes in $\omega_{q}$ on the transverse density profile of the beam [11]. In the present work, the effects of changing $\omega_{q}$ are explored experimentally. The transverse focusing frequency is proportional to $V_{0 \max } f^{-1}$ on PTSX so that there are two experimental parameters that can be changed to implement a variation in $\omega_{q}$. It is expected, within the smooth-focusing model, that only the change in $\omega_{q}$ is relevant, and not whether the change is due to changes in $V_{0 \max }$ or $f$.
Experiments on the transverse compression of plasmas in PTSX due to changes in $V_{0 \max }$ have been discussed by Chung et al. [20] and those results will be summarized here and discussed further in a broader context where they are part of a more complete discussion of variations in $\omega_{q}$. Experiments in which $f$ is varied in order to adiabatically compress the beam demonstrate that decreases in $f$ are equally well suited as increases in $V_{0 \max }$ for compressing the radial density profile and increasing the peak density of the plasma. For the plasmas used in these experiments with a normalized beam intensity $s=0.2, R_{b}=0.85 \mathrm{~cm}$, and $\mathrm{kT}=0.13 \mathrm{eV}$, the transverse compression may still be considered adiabatic even if the transition is made over only 4 lattice periods. Further, the peak density scales with the transverse focusing frequency as $n_{b}(0) \propto \omega_{q}$ as expected. However, the normalized beam intensity parameter decreases because $s \propto \omega_{q}^{-1}$ due to the dependence of $n_{b}(0)$ on $\omega_{q}$. Instantaneous changes in $\omega_{q}$ may still transversely compress the plasma, but they create a sudden beam mismatch that ultimately relaxes and increases the long-time transverse emittance (transverse temperature). It is this long-time state that is observed since PTSX measurements are averaged over hundreds of lattice periods. Finally, slow changes in the average frequency can be implemented as an example to demonstrate the flexibility of the PTSX facility and emphasize basic key points about beam stability.

\section{CHANGES IN LATTICE AMPLITUDE $\boldsymbol{V}_{\mathbf{0} \max }$}

Adiabatic changes in $\omega_{q}$ are made by letting $\omega_{q}$ vary according to

$$
\omega_{q}(t)=\omega_{q 1}+\frac{\omega_{q 0}-\omega_{q 1}}{2}\left[\tanh \frac{-\left(t-\tau_{1 / 2}\right)}{\tau / 2}+1\right],
$$

where $\omega_{q 0}$ is the initial value of $\omega_{q}, \omega_{q 1}$ is the final value of $\omega_{q}, \tau_{1 / 2}$ is the time where the transition is half complete, and $\tau$ is the time scale over which the transition is made. The product $\tau f$ is then approximately equal to the number of lattice periods over which the transition is made. When $\tau=0$, the transition is made instantaneously.

In Refs. [17,18,20], both adiabatic and instantaneous changes in $\omega_{q}$ of up to a factor of 2.2 are made by increasing the voltage waveform amplitude $V_{0 \max }$. Figure 6 of Ref. [20] is reproduced here as Fig. 3 to demonstrate the difference between instantaneous and adiabatic changes in $\omega_{q}$ and also demonstrate the good agreement between the experimental results and the theoretical models. For example, the results in Fig. 3 show that, for increases in $\omega_{q}$ of $50 \%$, instantaneous changes compress the plasma, but the peak density increases by only $30 \%$. The transverse emittance increases by $25 \%$. Further, the radial density profile shows that a large number of particles are transferred to a large radius forming a superGaussian tail, corresponding to excitation of a halo particle 

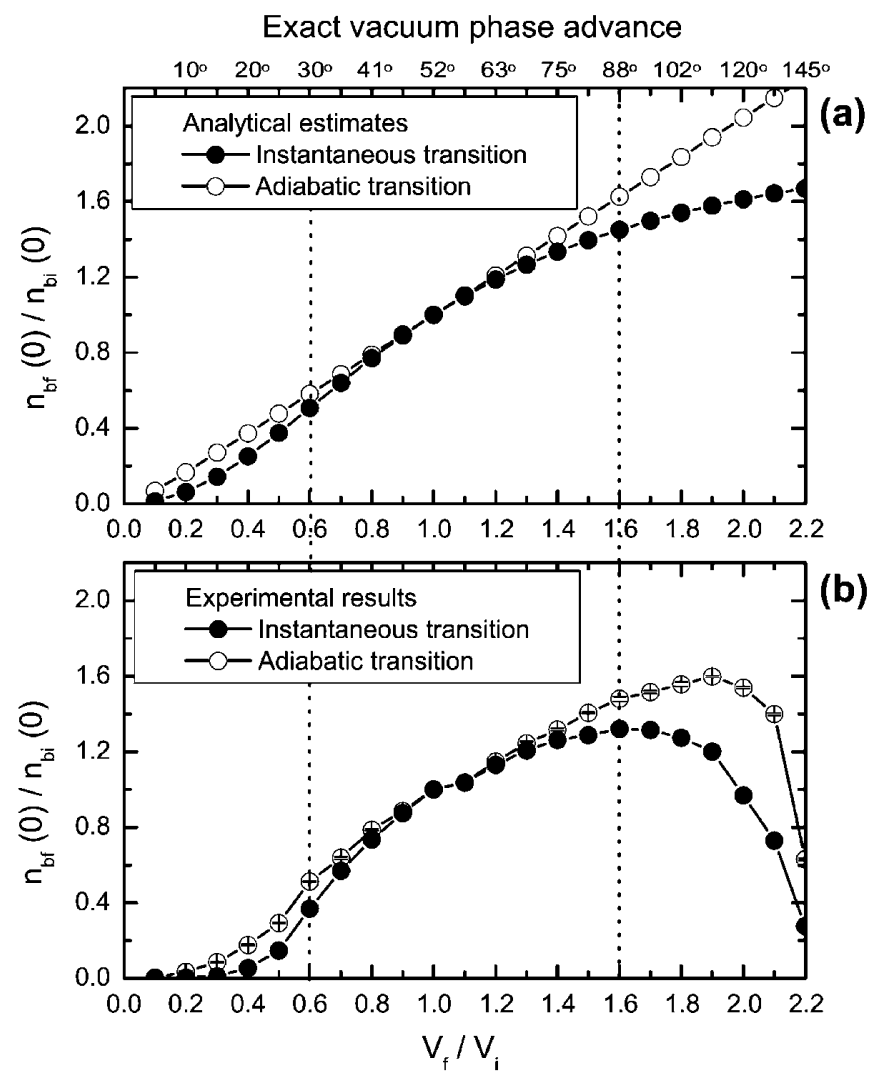

FIG. 3. Plots of the ratio of final to initial on-axis beam density $n_{b f}(0) / n_{b i}(0)$ for different values of $V_{f} / V_{i}$ with $f=60 \mathrm{kHz}$ [20]. The values $n_{b f}(0) / n_{b i}(0)$ are either (a) estimated from analytical theory, or (b) measured experimentally. Here, the initial exact vacuum phase advance $\sigma_{v i}$ is $52^{\circ}$, which corresponds to $\sigma_{v}^{\text {sf }}=49.8^{\circ}$. Both instantaneous (solid circles) and adiabatic (open circles) transitions are presented.

population. However, for an adiabatic increase in $\omega_{q}$ of $50 \%$ caused by an increase in $V_{0 \max }$, Fig. 3 shows that the peak density increases by $40 \%$. The emittance increases by only a few percent. In this case, the radial density profile remains approximately Gaussian. Over the range $0.6<$ $V_{f} / V_{i}<1.6$, the agreement between the experimental data and the analytical estimates is particularly good, confirming the scaling $n_{b}(0) \propto \omega_{q}$. Most interestingly, it was also shown in Refs. $[18,20]$ that the transition may be considered adiabatic when made over more than about four lattice periods. Making the transition more gradual than four lattice periods does not compress the charge bunch any further. Finally, it should be pointed out that particle-in-cell simulations made with the warp code [25] are in excellent agreement with the amplitude-change experiments described here $[11,18,20]$.

\section{CHANGES IN LATTICE PERIOD $f=1 / T$}

In this section, increases in $\omega_{q}$ created by decreasing the frequency $f=1 / T$ are considered. It is important to note here that voltage waveforms are applied to the PTSX electrodes by generating the waveform $V_{0 \max } \sin \phi(t)$, where $\phi$ is an arbitrary function of time, so that once the functional form of the instantaneous frequency $\dot{\phi}(t) / 2 \pi$ is specified, it must be integrated to obtain $\phi(t)$. To obtain a hyperbolic tangent transition of the instantaneous frequency, the required phase function is

$$
\begin{aligned}
\phi(t)= & 2 \pi \frac{f_{1}+f_{0}}{2} t+2 \pi \frac{f_{1}-f_{0}}{2} \frac{\tau}{2} \\
& \times \ln \left[\cosh \left(\frac{-\left(t-t_{1 / 2}\right)}{\tau / 2}\right)\right] .
\end{aligned}
$$

In Fig. 4, the final measured on-axis charge $Q(0)$, which is proportional to the peak plasma density, is plotted versus the number of initial lattice periods over which the increase in $\omega_{q}$ is made. The on-axis charge is $0.48 \mathrm{pC}$ corresponding to normalized beam intensity $s=0.2$, the rms radius is $0.85 \mathrm{~cm}$, and the temperature is $0.13 \mathrm{eV}$ before the charge bunch is compressed. Note that because the smooth-focusing vacuum phase $\sigma_{v}^{\text {sf }}$ advance scales like $V_{0 \max }^{1} f^{-2}, \sigma_{v}^{\text {sf }}$ increases more rapidly when the frequency is changed in order to compress the plasma than when the amplitude is changed. Given the operating parameters of the baseline case $\left(V_{0 \max }=150 \mathrm{~V}, f=60 \mathrm{kHz}\right), \omega_{q}$ cannot be increased as much compared to when $V_{0 \max }$ is increased, or else $\sigma_{v}^{\text {sf }}$ will exceed the single-particle orbit stability limit $\sigma_{v \text { critical }}^{\text {sf }}=115.6^{\circ}$ [14]. This is the value of the limit for the sinusoidal waveforms used in the present experiments and corresponds to the limit of the exact vacuum phase advance $\sigma_{v \text { critical }}=180^{\circ}$ [14]. Therefore, a maximum increase in $\omega_{q}$ of $33 \%$ is used.

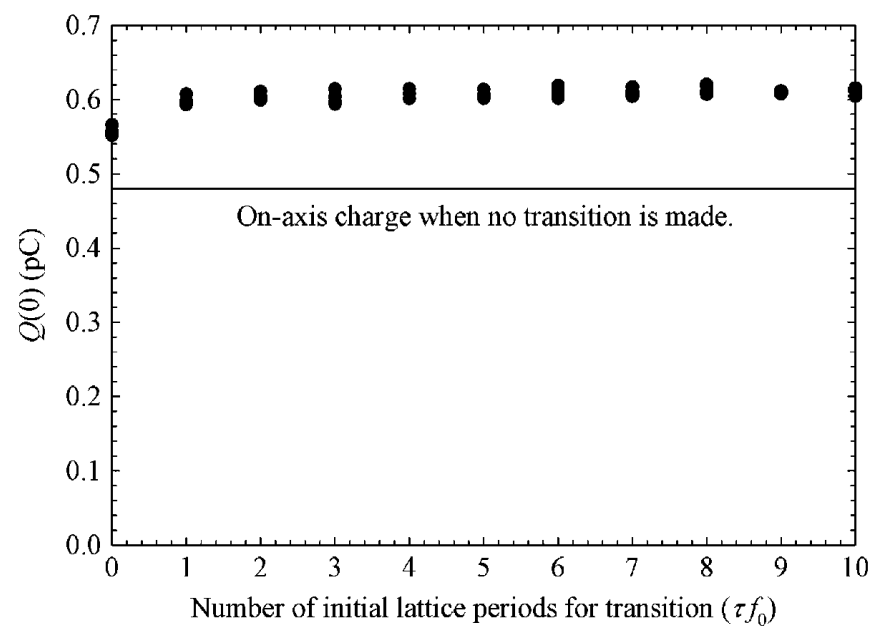

FIG. 4. The measured on-axis charge $Q(0)$, which is proportional to the peak plasma density, varies with the number of initial lattice periods over which the increase in $\omega_{q}$ is made. $Q(0)$ increases when the instantaneous frequency $\dot{\phi}(t) / 2 \pi$ is decreased from 60 to $45 \mathrm{kHz}$. An instantaneous change compresses the beam less and increases the emittance as compared to an adiabatic change that is made over two or more lattice periods. 


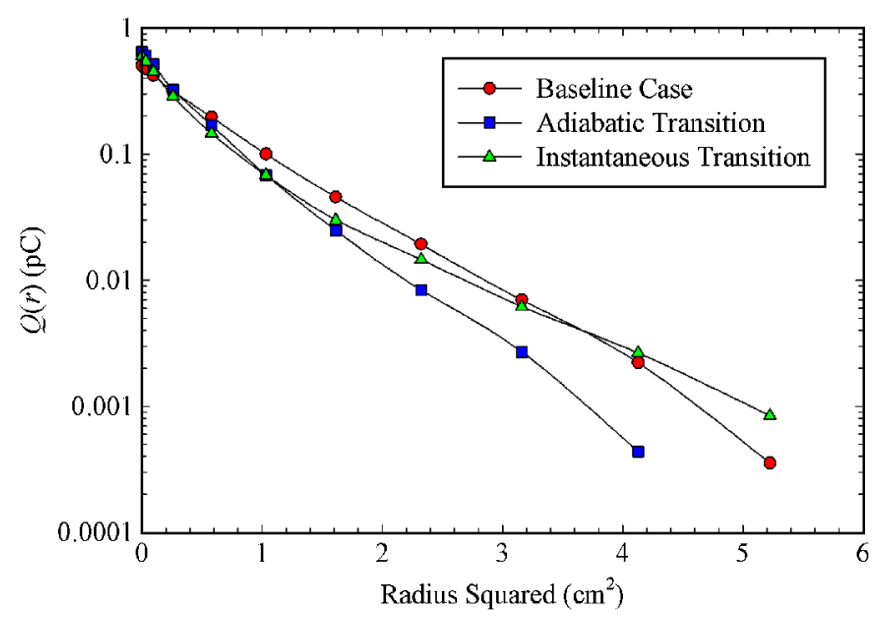

FIG. 5. (Color) The average radial density profile plotted versus $r^{2}$ is linear when the density profile is Gaussian. Both the baseline case and the adiabatically compressed case have radial density profiles that are Gaussian. In contrast, the instantaneously compressed case has a radial density profile in which some particles have been transferred to a larger radius, thereby producing a super-Gaussian tail.

An instantaneous decrease $(\tau=0)$ in the frequency from 60 to $45 \mathrm{kHz}$ compresses the plasma and raises the peak density by $17 \%$ while increasing the emittance by $24 \%$. If instead the transition is made over two or more lattice periods, the peak density is raised by $30 \%$ while the emittance does not change discernably. The radial density profiles in Fig. 5 show that an instantaneous change causes the creation of a distortion of the original Gaussian radial density profile, corresponding to excitation of a halo particle population. The radial density profile of the plasma after an adiabatic transition remains Gaussian. That the peak density scales linearly with $\omega_{q}$ and that there is minimal emittance growth when $\omega_{q}$ is increased adiabatically agree well with the case where $V_{0 \max }$ is changed while $f$ is held fixed. This confirms the notion that $\omega_{q}$ is the important control parameter in characterizing beam compression, as opposed to either $V_{0 \max }$ or $f$ separately. In both cases, the transition may be considered adiabatic when the transition is made over more than about four lattice periods. Note, however, that the change in the smooth-focusing phase advance is different in the two cases.

\section{ENVELOPE EQUATION MODEL COMPARISON}

It is informative to compare the experimental results to the solutions of the coupled transverse envelope equations where the initial condition is taken to be a $\mathrm{KV}$-equivalent matched-beam solution that has the same line density $N_{b}$, rms radius $R_{b}$, and effective transverse temperature $T$ as those measured [1]. This KV-equivalent beam represents a uniform-density charge distribution that has an elliptical

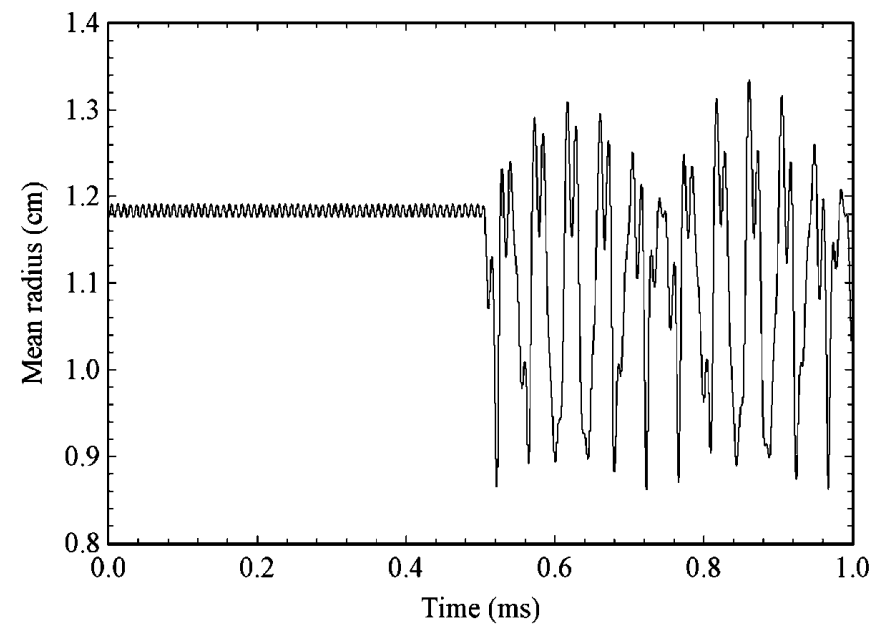

FIG. 6. The mean radius $\sqrt{a b}$ undergoes large-amplitude oscillations that do not correspond to a matched-beam solution of the coupled envelope equations when an instantaneous decrease in the frequency $\dot{\phi}(t) / 2 \pi$ from 60 to $45 \mathrm{kHz}$ to compress the charge bunch is made.

cross section with radii $a$ and $b$ in the transverse $x$ and $y$ directions. If the frequency $f$ is changed instantaneously from 60 to $45 \mathrm{kHz}$, then the mean radius $\sqrt{a b}$ decreases on average, but only by about $8 \%$, and the mean radius exhibits large oscillations as shown in Fig. 6. Further, the oscillations are not characteristic of a matched-beam solution. Note that a reduction in the mean radius of $8 \%$ corresponds to an increase in the peak density of $17 \%$ since $n_{b}(0) R_{b}^{2}=$ const. This corresponds to the experimentally observed increase in the peak density even though the emittance was taken to be constant in this calculation. The emittance is expected to, and indeed is observed to, increase in the experiment itself.

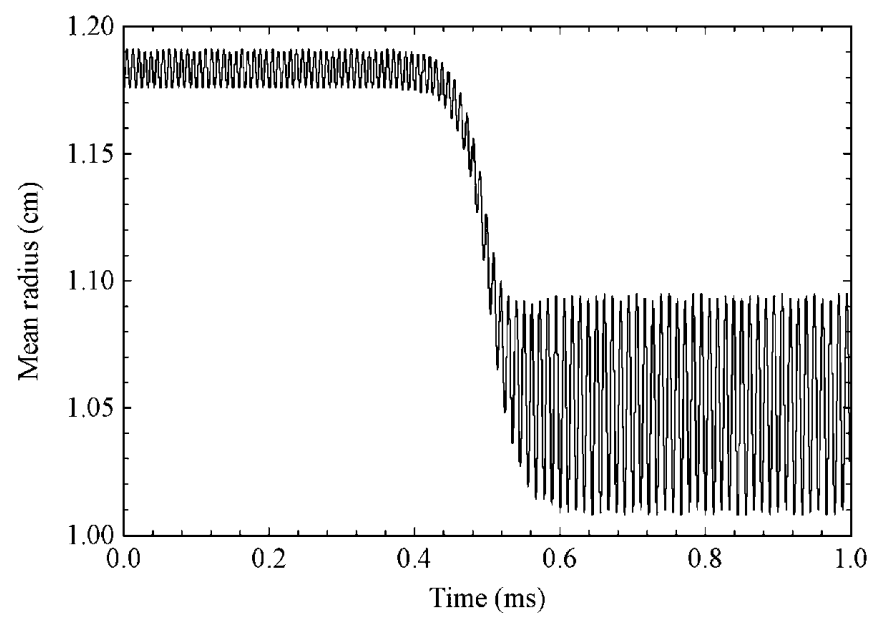

FIG. 7. The mean radius $\sqrt{a b}$ decreases as an adiabatic decrease in the frequency $\dot{\phi}(t) / 2 \pi$ from 60 to $45 \mathrm{kHz}$ made over 5 initial lattice periods, $\tau=5 \times(60 \mathrm{kHz})^{-1}$, compresses the charge bunch. The compression is more than in the instantaneous-change case, and the mean radius oscillations correspond to a beam that is still well matched. 
If the same $\mathrm{KV}$-equivalent beam is then subjected to a transverse compression administered over 5 initial lattice periods, then Fig. 7 shows that the final mean radius is smaller by $12 \%$ which is consistent with the observed increase in peak density of $30 \%$ mentioned above, since $1.12^{2}=1.25$. After the compression, the oscillations in the mean radius are much smaller than in the instantaneouschange case, and the beam remains well matched after the adiabatic transition.

\section{NONMONOTONIC VARIATION OF $\boldsymbol{\omega}_{q}$}

Having demonstrated that adiabatic, monotonic changes in the transverse focusing frequency $\omega_{q}$ can transversely compress the plasma when applied over as few as four lattice periods, regardless of whether the change is made by changing the lattice field-strength or the periodicity, nonmonotonic changes in $\omega_{q}$ are now considered. The applied voltage waveforms considered in this section allow the effects of varying $\omega_{q}(t)$ to be explored. The experimental results presented here provide a good example of the flexibility that the PTSX facility possesses, through the arbitrary voltage waveform $V(t)$, to simulate a wide variety of magnetic alternating-gradient transport system configurations.

The specific form of $\omega_{q}(t)$ that is considered is motivated by noting the difference between the instantaneous, or actual frequency $\dot{\phi}(t) / 2 \pi$ of the applied voltage waveform $V(t)=V_{0 \max } \sin \phi(t)$ and the average frequency $\phi / 2 \pi t$. In contrast to what was considered in the previous sections, if it is now required that the average frequency be prescribed by a hyperbolic tangent function in time, then the instantaneous frequency acquires a nonmonotonic form; therefore, so do $\omega_{q}(t)$ and $\sigma_{v}^{\mathrm{sf}}(t)$. The phase function assumes the form

$$
\phi(t)=2 \pi f_{1} t+\pi\left(f_{0}-f_{1}\right) t\left[\tanh \frac{-\left(t-t_{1 / 2}\right)}{\tau / 2}+1\right] .
$$

Both $\phi / 2 \pi t$ and $\dot{\phi}(t) / 2 \pi$ are plotted in Fig. 8 for the case where the initial frequency is $60 \mathrm{kHz}$, the final frequency is $50 \mathrm{kHz}$, and the transition is made over $1 \mathrm{~ms}$. In this case, $\tau=21.35 f_{0}^{-1}$ and $\tau_{1 / 2}=0.5 \mathrm{~ms}$.

Figure 9 shows the results of several experiments where the initial frequency $f_{0}$ is $60 \mathrm{kHz}$, and the final frequency $f_{1}$ ranges from 55 to $47 \mathrm{kHz}$. The on-axis charge is plotted versus the number of initial lattice periods over which the frequency change is applied. Depending on the final frequency, there is a threshold value $\tau_{c}\left(f_{1}\right)$ of the transition time below which transverse confinement is lost. For the cases where $f_{1}$ is 55 and $50 \mathrm{kHz}$, data were also taken for $\tau=0$, where the transitions are instantaneous and it is found that the plasma is not lost. Finally, when $\tau>\tau_{c}$ so that the transition is sufficiently gradual, the long-time value of the on-axis charge scales linearly with $f_{1} / f_{0}$ as expected. It is found that the plasma will adjust its shape to

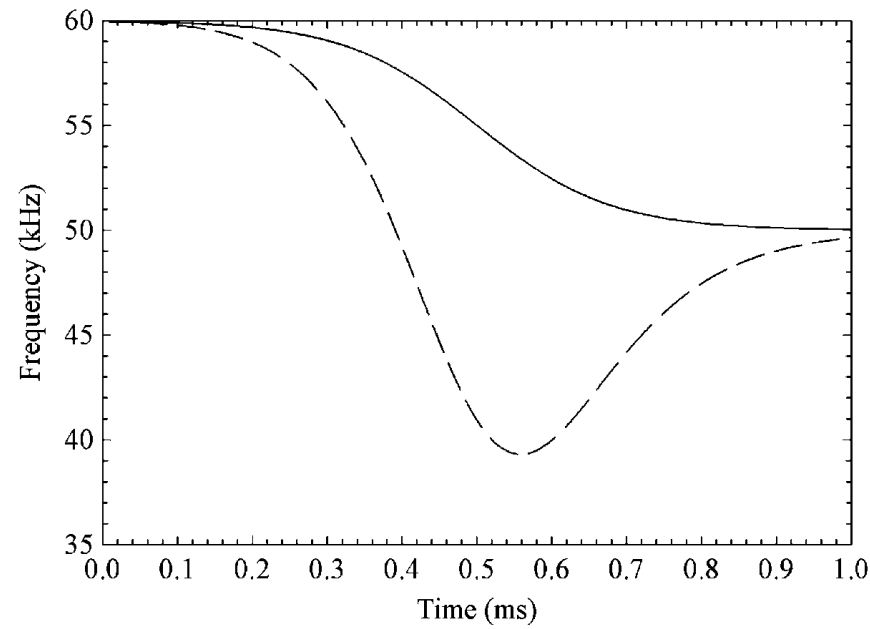

FIG. 8. If the average frequency $\phi(t) / 2 \pi t$ (solid) decreases like the hyperbolic tangent from 60 to $50 \mathrm{kHz}$, then the instantaneous frequency $\dot{\phi}(t) / 2 \pi$ (dashed) also transitions from $f_{0}$ to $f_{1}$, but undershoots. In this case, both the transverse focusing frequency and the phase advance overshoot.

maintain global force balance as long as $\omega_{q}$ is varied sufficiently slowly. For completeness, a data set is displayed in Fig. 10 in which $f_{1}=90 \mathrm{kHz}$ so that the charge bunch is allowed to expand. In this case, the transverse confinement is never completely lost, in contrast with the results shown in Fig. 9. Although, when the transition is made more rapidly than approximately 15 initial lattice periods, the final on-axis charge is smaller than when the transition is made more gradually.

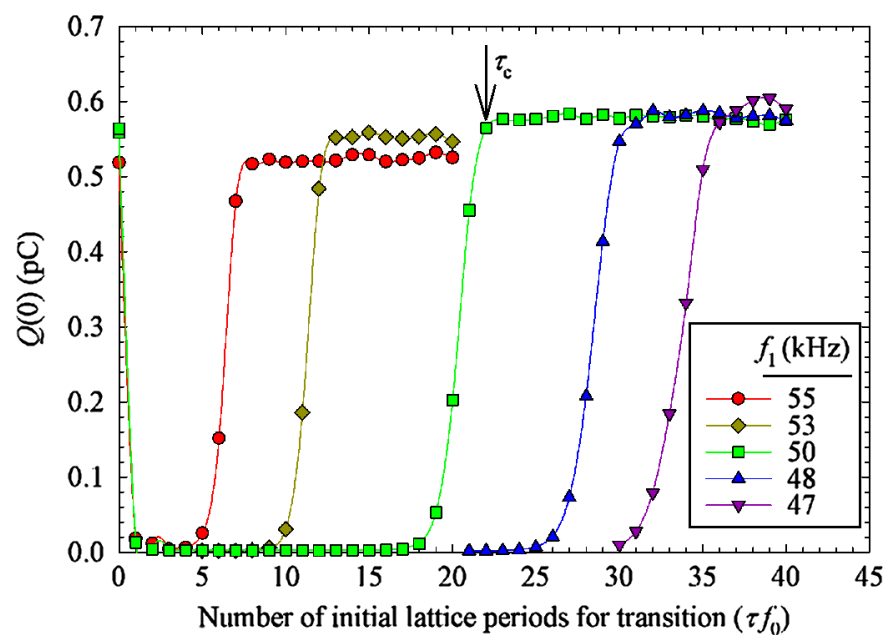

FIG. 9. (Color) The measured on-axis charge $Q(0)$, which is proportional to the peak plasma density, varies with the number of initial lattice periods over which the increase in $\omega_{q}$ is made. Adiabatic decreases in the average frequency $\phi(t) / 2 \pi t$ compress the plasma and raise the on-axis charge $Q(0)$ only if the transition time $\tau$ is greater than some critical time $\tau_{c}$. Otherwise, plasma confinement is lost. The exception is if the decrease in frequency is made instantaneously. 


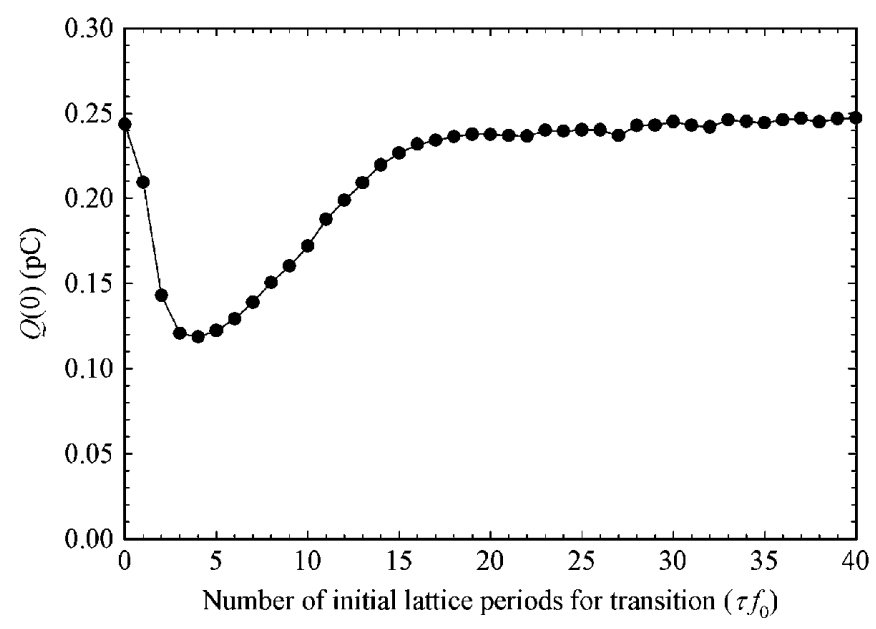

FIG. 10. The measured on-axis charge $Q(0)$, which is proportional to the peak plasma density, is plotted versus the number of initial lattice periods over which the decrease in $\omega_{q}$ is made. If the average frequency $\phi(t) / 2 \pi t$ is increased from 60 to $90 \mathrm{kHz}$, then the plasma is allowed to expand, thus reducing the on-axis charge $Q(0)$. If the transition time $\tau$ is less than about 15 initial lattice periods, then there is a further expansion of the plasma. As the transition time approaches zero and the transition becomes instantaneous, the increased expansion disappears.

These results can be understood by considering what is happening to the instantaneous frequency during the transition. Figure 8 shows how both the instantaneous and average frequencies vary during the transition to a lower frequency. When the average frequency assumes the form of the hyperbolic tangent function, the instantaneous frequency undershoots by an amount that depends on the difference between the initial and final frequencies and the transition time. If the minimum instantaneous frequency is small enough so that the smooth-focusing vac-

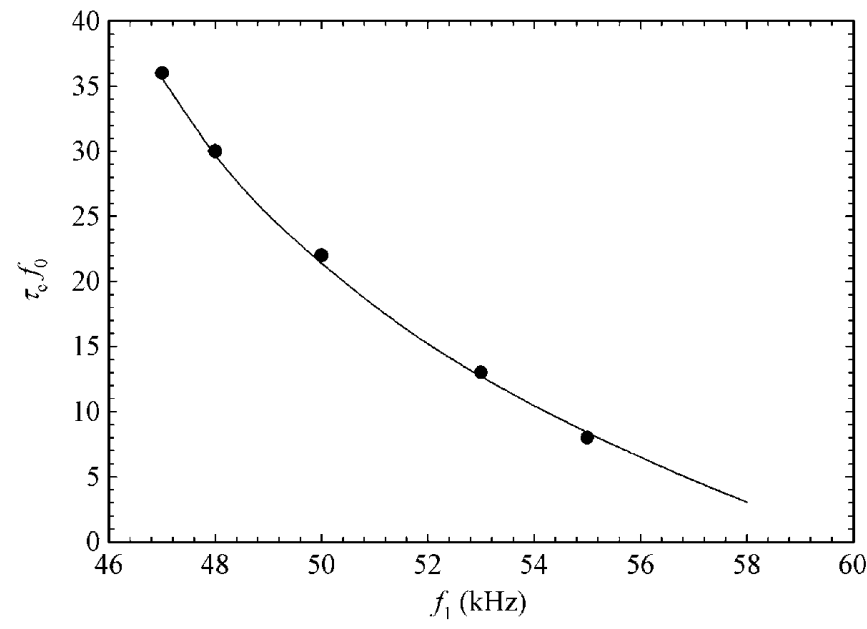

FIG. 11. The values of $\tau_{c}$ extracted from the data in Fig. 9 are plotted together with a curve derived from solving the equation that results from demanding that the maximum smooth-focusing vacuum phase advance that occurs during the transition equal the maximum value for single-particle orbit stability $115.6^{\circ}$. uum phase advance exceeds the single-particle orbit stability limit, then transverse confinement will be lost. The transcendental equation that results from writing down the condition that the maximum smooth-focusing vacuum phase advance equals $\sigma_{v \text { critical }}^{\text {sf }}$ can be solved numerically to find $\tau_{c}$ for a given $f_{0}$ and $f_{1}$. For the data in Fig. $9 f_{0}=60 \mathrm{kHz}$ and so $\tau_{c}\left(f_{1}\right)$ can be computed and compared to the measured values of $\tau_{c}$ as in Fig. 11. The excellent agreement confirms that transverse confinement of the charge bunch is indeed lost because the singleparticle orbits become unstable.

In the case where the average frequency is changed instantaneously, the smooth-focusing vacuum phase advance does not exceed $\sigma_{v \text { critical }}^{\text {sf }}$ at any time, and the charge bunch remains confined. Transverse compression is achieved, but with substantial emittance growth. When $\omega_{q}$ is decreased by increasing the final frequency as in Fig. 10, $\omega_{q}$ and $\sigma_{v}^{\text {sf }}$ undershoot during the transition and the single-particle orbits remain stable. However, when the transition is too rapid, the undershoot in $\omega_{q}$ allows the charge bunch to expand and the particles to be lost to the wall, resulting in a decreased on-axis charge after the transition in complete.

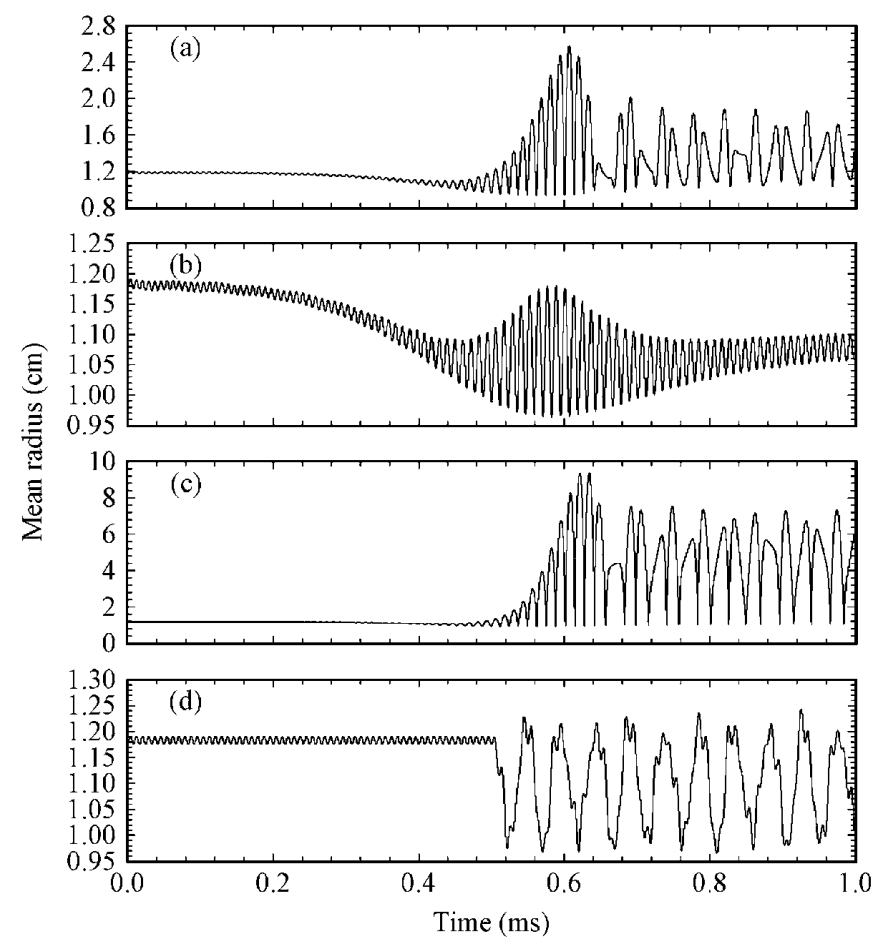

FIG. 12. The evolution of the mean radius $\sqrt{a b}$ is plotted for four different values of the transition time scale $\tau$. (a) When $\tau=$ $\tau_{c}$, the mean radius undergoes large-amplitude oscillations and the final beam is not well matched. (b) When $\tau \gg \tau_{c}\left(26 f_{0}^{-1}\right.$ here), the charge bunch is able to follow the changing transverse focusing frequency and the final state is well matched. (c) As $\tau$ is decreased further from $\tau_{c}$, the mean radius grows to the wall radius $r_{w}=10 \mathrm{~cm}$. (d) Instantaneous changes compress the plasma, but leave large residual oscillations of the mean radius. 
Similar results are seen in the solutions of the coupled transverse envelope equations. For the case where $f_{0}=$ $60 \mathrm{kHz}, f_{1}=50 \mathrm{kHz}$, and $\tau=\tau_{c}=21.35 f_{0}^{-1}$, the mean radius is observed to momentarily increase before settling to an unmatched oscillation about a larger mean radius [Fig. 12(a)]. It is not until $\tau=26 f_{0}^{-1}$ that the long-time oscillations in the mean radius correspond to a matchedbeam solution [Fig. 12(b)]. Conversely, once $\tau<19.9 f_{0}^{-1}$, the mean radius exceeds the $10 \mathrm{~cm}$ wall radius of the machine during a portion of the transition [Fig. 12(c)]. Finally, as in Sec. VII, an instantaneous decrease in the frequency causes the mean radius to oscillate about a smaller long-time value, demonstrating transverse compression, albeit with a beam mismatch that ultimately leads to an increase in emittance [Fig. 12(d)].

\section{CONCLUSIONS}

The transverse compression of a charged particle beam moving through a magnetic alternating-gradient transport system can focus the beam to a small spot size in order to deliver more particle flux and energy to the target. It is important to determine how few lattice periods are needed to make such a transverse compression while maintaining a reasonably well-matched-beam envelope. The results of experiments performed with the flexible PTSX facility show that, for moderately intense beams with normalized beam intensity parameter $s=0.2\left(\nu / \nu_{0} \sim 0.9\right)$, transverse compression can be affected by changes in the smoothfocusing frequency $\omega_{q}$ over only four lattice periods. The changes in $\omega_{q}$ can be made by using the arbitrary waveform voltage $V(t)$ to change either the field strength or the lattice periodicity, although the final smooth-focusing vacuum phase advance depends on whether $V_{0 \max }$ or $f$ is changed. As long as the transition is made sufficiently gradual, even nonmonotonic changes in $\omega_{q}$ can be made.

\section{ACKNOWLEDGMENTS}

This research was supported by the U.S. Department of Energy. It is a pleasure to acknowledge the benefit of useful discussions with Mikhail Dorf.

[1] R. C. Davidson and H. Qin, Physics of Intense Charged Particle Beams in High Intensity Accelerators (World Scientific, Singapore, 2001).

[2] M. Reiser, Theory and Design of Charged Particle Beams (Wiley, New York, 1994).

[3] A. W. Chao, Physics of Collective Beam Instabilities in High Energy Accelerators (Wiley, New York, 1993).
[4] P. G. O'Shea et al., Nucl. Instrum. Methods Phys. Res., Sect. A 464, 646 (2001).

[5] N. Kjærgaard and M. Drewsen, Phys. Plasmas 8, 1371 (2001).

[6] A. B. Sefkow and R. C. Davidson, Phys. Rev. ST Accel. Beams 9, 090101 (2006).

[7] S. M. Lund and B. Bukh, Phys. Rev. ST Accel. Beams 7, 024801 (2004).

[8] L. K. Spentzouris, J.-F. Ostiguy, and P. L. Colestock, Phys. Rev. Lett. 76, 620 (1996).

[9] D. Neuffer, E. Colton, D. Fitzgerald, T. Hardek, R. Hutson, R. Macek, M. Plum, H. Thiessen, and T.-S. Wang, Nucl. Instrum. Methods Phys. Res., Sect. A 321, 1 (1992).

[10] J. Byrd, A. Chao, S. Heifets, M. Minty, T. O. Raubenheimer, J. Seeman, G. Stupakov, J. Thomson, and F. Zimmerman, Phys. Rev. Lett. 79, 79 (1997).

[11] M. Dorf, R. C. Davidson, and E. A. Startsev, Phys. Rev. ST Accel. Beams 9, 034202 (2006).

[12] R. C. Davidson, H. Qin, and G. Shvets, Phys. Plasmas 7, 1020 (2000).

[13] E. P. Gilson, R. C. Davidson, P. C. Efthimion, R. Majeski, and H. Qin, in Proceedings of the 2003 Particle Accelerator Conference (IEEE Catalog No. 03CH37423C, 2003), p. 2655.

[14] E. P. Gilson, R. C. Davidson, P. C. Efthimion, R. Majeski, and E. A. Startsev, AIP Conf. Proc. 692, 211 (2003).

[15] E. P. Gilson, R.C. Davidson, P.C. Efthimion, and R. Majeski, Phys. Rev. Lett. 92, 155002 (2004).

[16] E. P. Gilson, M. Chung, R. C. Davidson, P.C. Efthimion, R. Majeski, and E. A. Startsev, Nucl. Instrum. Methods Phys. Res., Sect. A 544, 171 (2005).

[17] E. P. Gilson, M. Chung, R. C. Davidson, M. Dorf, P. C. Efthimion, and R. Majeski, Phys. Plasmas 13, 056705 (2006).

[18] E. P. Gilson, M. Chung, R. C. Davidson, M. Dorf, D. Grote, P.C. Efthimion, R. Majeski, and E. A. Startsev, Nucl. Instrum. Methods Phys. Res., Sect. A 577, 117 (2007).

[19] M. Chung, E. P. Gilson, M. Dorf, R. C. Davidson, P. C. Efthimion, and R. Majeski, Phys. Rev. ST Accel. Beams 10, 014202 (2007).

[20] M. Chung, E. P. Gilson, M. Dorf, R. C. Davidson, P. C. Efthimion, and R. Majeski, Phys. Rev. ST Accel. Beams 10, 064202 (2007).

[21] H. Okamoto and H. Tanaka, Nucl. Instrum. Methods Phys. Res., Sect. A 437, 178 (1999).

[22] N. Kjærgaard, K. Mølhave, and M. Drewsen, Phys. Rev. E 66, 015401 (2002).

[23] R. Takai, H. Enokizono, K. Ito, Y. Mizuno, K. Okabe, and H. Okamoto, Jpn. J. Appl. Phys. 45, 5332 (2006).

[24] W. Paul and H. Steinwedel, Z. Naturforsch. A 8, 448 (1953).

[25] A. Friedman, D. P. Grote, and I. Haber, Phys. Fluids B 4, 2203 (1992). 